\title{
Early hematopoiesis inhibition under chronic radiation exposure in humans
}

\author{
Alexander V. Akleyev • Igor V. Akushevich • Georgy P. Dimov • \\ Galina A. Veremeyeva - Tatyana A. Varfolomeyeva \\ Svetlana V. Ukraintseva $\cdot$ Anatoly I. Yashin
}

Received: 6 July 2009/Accepted: 4 February 2010/Published online: 26 March 2010

(C) Springer-Verlag 2010

\begin{abstract}
The major goal of this study was to identify and quantitatively describe the association between the characteristics of chronic (low-dose rate) exposure to (low LET) ionizing radiation and cellularity of peripheral blood cell lines. About 3,200 hemograms (i.e., spectra of blood counts) obtained over the years of maximal exposure to ionizing radiation (1950-1956) for inhabitants of the Techa River were used in analyses. The mean cumulative red bone marrow dose (with standard errors), calculated using Techa River Dosimetry System-2000, was $333.6 \pm$ $4.6 \mathrm{mGy}(\mathrm{SD}=259.9 \mathrm{mGy}, \max =1151 \mathrm{mGy})$ to the year 1956. The statistical approach included both empirical methods for estimating frequencies of cytopenic states of the investigated blood cell lines (e.g. neutrophile, platelets, erythrocyte, etc.), and regression methods, including generalized linear models and logistic regressions which allowed taking into account confounding factors (e.g., attained age, age at maximal exposure, presence of concomitant diseases, and demographic characteristics). The results of the analyses demonstrated hematopoiesis inhibition manifested by a decrease in peripheral blood cellularity and an increase in the frequency of cytopenia in all blood cell lines (leukocytes, including lymphocytes, monocytes, neutrophiles, as well as platelets and erythrocytes). The intensity of hematopoiesis inhibition in the
\end{abstract}

A. V. Akleyev · G. P. Dimov ( $₫)$ · G. A. Veremeyeva ·

T. A. Varfolomeyeva

Clinical Department,

Urals Research Center for Radiation Medicine,

Chelyabinsk, Russia

e-mail: dimov@urcrm.chel.su; first-aid03@yandex.ru

I. V. Akushevich · S. V. Ukraintseva · A. I. Yashin Center for Population Health and Aging, Duke University,

Durham, NC, USA period of maximal exposures is determined by the combined influence of the dose rate and cumulative dose. The contribution of specific confounding factors was quantified and shown to be much less important than dose characteristics. The best predictor among dose characteristics was identified for each blood cell line. A 2-fold increase in dose rate is assumed to be a characteristic of radiosensitivity and a quantitative characteristic of the effect.

\section{Introduction}

Hematopoietic tissue is one of the most radiosensitive tissues of the human body. High sensitivity of the hematopoietic system to acute and chronic radiation exposure has been demonstrated in numerous experiments on animals (Seed 1996; Seed et al. 2002a, b; Shvedov and Akleyev 2001; Muksinova and Mushkacheva 1990). The status of hematopoiesis determines the outcomes of acute radiation syndrome and is critical for the patient's survival (Bond et al. 1965; Guskova and Baysogolov 1971). In the study by Fliedner et al. (2002a) the pathophysiology of the chronic radiation exposure syndrome both for human beings and for a number of animal species has been reviewed and discussed in details. It should be noted that reactions of human hematopoiesis to chronic low intensive exposure has been studied insufficiently as yet.

A characteristic feature of chronic radiation exposure is its prolonged damaging effect on the cell structures (nucleus, cell membrane, etc.) accompanied by simultaneous adaptation processes. Mechanisms of adaptation to chronic radiation exposure in different tissues have some specific features determined by dependence on the specificity of structure and function of cells and organization of tissue (Paranich et al. 2001). 
The reaction of tissues to radiation is a threshold effect. It is considered that induction of the same deterministic effects of chronic exposure requires higher cumulative doses of radiation than those of acute exposure (ICRP 1991). Different species of experimental animals can survive high doses of chronic radiation which exceed absolute lethal doses of acute radiation without impairment of function of exposed tissues, organs, and the whole organism (Yagunov et al. 1998). It is important to differentiate between dose thresholds that lead to adaptive reactions of cells and tissues and those bringing about changes in the structure and function of tissue.

Tissue reaction to exposure is an integral result of all different cell responses in exposed tissue and system to which this tissue belongs. The reaction of hematopoiesis includes responses of red bone marrow (RBM), hematopoietic cells, peripheral blood and other organs as well as intercellular matrix, endothelium of blood vessels, fibroblasts, macrophages, and others. The mechanism of tolerance of tissue to chronic radiation has a very complex origin and is connected with a complex of subcellular, cellular, tissue, organ, and even organism's adaptive reactions (Baraboǐ and Oleǐnik 1999).

The mechanisms of the hematopoietic reaction to chronic radiation exposure have been investigated more extensively in animals. A complex rearrangement takes place in hematopoietic tissue under chronic low-dose rate and low LET radiation exposure with involvement of different mechanisms of adaptation. Hematopoiesis can maintain a sufficient level of blood cell functioning due to increased cell production associated with shortening of cell cycle and maturation period (Grygoryev et al. 1986), intensification of proliferation activity of early cell forms (stem and progenitor cells) (Muksinova and Mushkacheva 1990), and development of extramedular hematopoiesis (Fliedner et al. 2002b). Besides, radioadapted animals have displayed an increased repair of sublethal damage in bone marrow progenitors (Seed et al. 2002b).

The number and quality of surviving hematopoietic stem cells are critical for hematopoietic recovery. Reducible damage (that is possible spontaneous hematopoietic recovery) is observed if more than $2 \%$ of stem and progenitor cells stay intact for replication and differentiation. If their level decreases below the critical one, the hematopoietic system can be exhausted due to the deficiency of replacement of stem cells for proliferation and differentiation (Fliedner et al. 2002b).

Significant individual variability of tissue reaction is manifested by animals in chronic exposure experiments. This variability is caused by different peculiarities of the organism, including its capacity for repair of radiation damage (Novoselova and Safonova 1994).

Hematopoiesis inhibition is a key link in the pathogenesis of early effects of chronic radiation exposure within a definite dose range. These effects are determined by the loss of stem and committed progenitor cells resulting in a decrease in peripheral blood counts (Fliedner et al. 2002b). Data obtained from previous studies of the Techa River population show a decrease in thrombocyte, neutrophile, and lymphocyte counts at RBM dose rates of 0.3-0.5 Gy/ year, or higher. These changes persisted for several years (Akleyev et al. 1999; Akleyev and Varfolomeyeva 2007). Under the conditions of chronic radiation exposure at dose rates over $4.5 \mathrm{~Gy} / \mathrm{year}$ and a cumulative dose over $8 \mathrm{~Gy}$, Mayak PA workers developed fatal hypoplasia of RBM with depression of all blood cell lines (Okladnikova 2001). Moreover, many parameters of early radiation-related hematopoiesis abnormalities have not yet been elucidated. The duration of the latent period, radiosensitivity of each blood cell line, and their dose and dose-rate dependence are still less known.

The aim of the present study is to estimate retrospectively the influence of the RBM cumulative dose, dose rate and different modifying factors on the parameters of peripheral blood cellularity under chronic low-dose-rate radiation exposure in humans, and to develop an approach to quantitative estimation of the effect.

\section{Materials and methods}

Description of population and clinical methods

Investigation of peripheral blood cellularity was conducted for the Techa riverside population which had been exposed to long-lived radionuclides for several decades as a result of failures in the technological processes at the Mayak plutonium facility, Southern Urals, Russia. A major source of environmental contamination was the discharge of about $10^{17} \mathrm{~Bq}$ of liquid wastes into the Techa River in 19491956 (Degteva et al. 2006).

Members of the Techa River Cohort (TRC) numbering nearly 30,000 residents of the Techa riverside area were exposed to a complex mixture of radionuclides, largely ${ }^{90} \mathrm{Sr}$ and ${ }^{137} \mathrm{Cs}$. The system of regular follow-up allows ascertainment of vital status, cause of death, and cancer incidence. With over 50 years of follow-up, the TRC now provides a valuable opportunity to study a wide range of health effects, both early and late, associated with protracted internal and external radiation exposures. The wide range of doses allows analysis of the nature of the doseresponse relationship based on internal comparisons (Kossenko et al. 2005).

The study group was comprised of exposed people who had been followed up by the URCRM researches from 1950 to 1956 (the period of maximal radiation exposure), and whose RBM exposure doses had been estimated. The 
results of the first complete blood count were included in the analysis. The study cohort included 3,152 people, 1,182 $(37.5 \%)$ men and $1,970(62.5 \%)$ women. The health status information for these persons was retrieved from the archives and the databases maintained at the URCRM.

Mean dose rate of RBM exposure to IR (with standard errors) for the period 1950-1956, calculated using Techa River Dosimetry System-2000 (TRDS-2000), was $42.8 \pm 0.99 \mathrm{mGy} / \mathrm{y} \quad(\mathrm{SD}=54.2 \mathrm{mGy} / \mathrm{y}, \quad \max =305.4$ $\mathrm{mGy} / \mathrm{y})$. Mean dose of $\gamma$-irradiation was $14.6 \pm 0.71 \mathrm{mGy} / \mathrm{y}$, and mean dose rate of internal exposure due to incorporated ${ }^{90} \mathrm{Sr}$ was $28.2 \pm 0.40 \mathrm{mGy} / \mathrm{y}$. The dose-reconstruction process is extensively based on the results of postmortem measurements of ${ }^{90} \mathrm{Sr}$ concentration in bone samples obtained in 1951-1993, the results of in vivo measurements of surface-beta activity of teeth using tooth-beta counters (1959-1997), and on measurements of ${ }^{137} \mathrm{Cs}$ and ${ }^{90} \mathrm{Sr}$ body burden in a whole body counter (1974-1997). Mean cumulative $\mathrm{RBM}$ dose in 1956 was $333.6 \pm 4.6 \mathrm{mGy}$ $(\mathrm{SD}=259.9 \mathrm{mGy}, \max =1151 \mathrm{mGy}$ ) (Napier et al. 2001; Degteva et al. 2006, 2007). The internal exposure was a determinative factor for about $80 \%$ of the riverside population (lower and middle reaches of the Techa). The residents of the upper reaches were exposed both internally and externally, and the levels of each type of exposure were comparable (Degteva et al. 2007; Anspaugh et al. 2006).

The following parameters of peripheral blood cellularity expressed in absolute values were selected for inclusion in the study: leukocyte (the concentration of all nucleated blood cells), neutrophile, lymphocyte, monocyte, platelet, and erythrocyte counts. The following values (expressed in $10^{9}$ cells/L) were used as the low limits of the norm (Sokolov and Gribova 1972): 4.0 for leukocytes, 2.0 for neutrophiles, 1.3 for lymphocytes, 2.0 for monocytes, 180 (men) and (150 (women) for thrombocytes, and 4.0 (men) and 3.7 (women) for erythrocytes in $10^{12}$ cells/L. We understand by cytopenia a condition characterized by a reduction or a lack of cellular elements in the circulating blood and by a lower-than-normal number of blood cells.

\section{Statistical methods}

The statistical methods used in this study are based on empirical estimates of the frequencies of cytopenic states for all blood cell lines of interest. The resultant data were analyzed subsequently using generalized linear models and logistic regression (McCullagh and Nelder 1989).

In generalized linear models, the response (i.e., blood counts) is assumed to have a probability distribution of the generalized exponential form, which includes a number of standard distributions, such as gamma and normal distributions. These distributions were used for description of blood count data, e.g., the data presented in (Sokolov and Gribova 1972) demonstrate that in normal conditions the parameters of cellularity of blood cell lines are characterized by normal distribution. Our analyses have shown that each of the blood cell lines under study can be characterized using one of these distributions. These two distributions were investigated for each blood cell line assuming that the mean is expressed in terms of linear combination of three predictors: dose rate at the year of measurement, cumulative dose, and attained age. The best model was selected by using the likelihood ratio test (Cox and Hinkley 1974). It was found that gamma distribution is the best model to describe the distribution shape for all blood cell lines (except erythrocytes) while the distribution of erythrocytes takes a normal shape. Only age and dose rate were found to significantly contribute to the specific blood count model. Thus, the model for all lines except erythrocytes (denoted by $y$ ) is described by the density of $\gamma$-distribution $f \gamma(y, \mu, v)$ specified in our case as

$f_{\gamma}(y, \mu, v)=\frac{1}{\Gamma(v) y}\left(\frac{y v}{\mu}\right)^{v} \exp \left(-\frac{y v}{\mu}\right)$

where $v$ is the scale parameter, and mean $\mu$ and variance $V$ of $y$ are further specified as

$\mu=u_{\alpha}+\alpha_{r} r_{d}+\alpha_{a}$ age, $\quad V=\mu^{2} / v$

where $u$ is an intercept, and $\alpha_{r}$ and $\alpha_{a}$ are parameters which define the contribution of annual dose rate and age to the mean of respective blood count. The distribution of erythrocytes is normal, where standard deviation $\sigma$ plays the role of the scale parameter, $V=\sigma^{2}$, and mean is specified as $\mu=u_{\alpha}+\alpha_{r} r_{d}$.

The logistic regression is used to describe the observed frequencies in terms of predictors, such as dose characteristics, age, and health state represented by a set of 102 diseases. Thus, a dependent variable in the logistic regression is the binary variable describing whether an individual had developed a respective cytopenic state as of date of measurement. Let $p_{L}$ be the response probability of being healthy, i.e., not to develop a cytopenic state in blood cell line $L$. Note, this choice to define the response probabilities help to keep the same sign of dose effects in two supplementing analyses based on the blood count distributions and frequencies of cytopenic states. The frequencies of cytopenic states are empirical estimates of the probabilities $1-p_{L}$. They are calculated in selected regions of dose characteristics, e.g., dose rate, $f_{L}=N_{c}\left(r_{d}\right) / N\left(r_{d}\right)$, where $N\left(r_{d}\right)$ is the total number of individuals exposed at dose rates within the range of interest, and $N\left(r_{d}\right)$ is the number of such individuals with the measured blood count below the norm.

There are three stages of analyses involving the logistic regression model: (1) description of the frequency in terms 
of classic radiobiological predictors, as it was performed above for analysis of the shape of blood count distributions, (2) search of the best predictors among dose characteristics and analysis of the confounding contributions of morbidity and nonradiation factors, and (3) using an established model for calculation of dose characteristics capable to describe radiosensitivity and possible threshold effects of certain population groups under chronic exposure to IR.

Similar to the case of modeling the shape of blood count distribution, the same three predictors (i.e., age, dose rate, cumulative dose) were used to describe the dependences influencing the frequency of cytopenic states. Note that logistic regression is also a particular case of generalized linear model. In this case Bernoulli distribution is assumed for individual measurement (presence or absence of the cytopenic state) and the mean value is linked to linear predictors through logit function (Hosmer and Lemeshow 2000).

The general formula for logistic regression analysis involving multiple dose characteristics and contributions of specific diseases is as follows:

$$
\begin{aligned}
\log \operatorname{it}\left(p_{L}\right) & =\log \frac{p_{L}}{1-p_{L}} \\
& =u+\beta_{r} r_{d}+\cdots+\beta_{c d} D_{c}+\beta_{a} \text { Age }+\sum_{i=1}^{102} \beta_{i} \cdot 1_{i} .
\end{aligned}
$$

Here, $r_{d}$ and $D_{c}$ are the dose rate and cumulative dose, respectively. Dots between these two contributions mean that we investigate various dose characteristics as predictors of the frequencies trying to select the best one. The sum over $i$ reflects contributions of 102 selected disease, in which $1_{i}$ is an $i$ th disease indicator. $\beta$ 's are regression parameters to be estimated. The SAS software Proc Logistic is used for parameter estimation through maximizing a likelihood utilizing individual information on dose characteristics and occurrence of cytopenia and other diseases. We use the stepwise technique for the estimation of the regression model (Hosmer and Lemeshow 2000). It means that the model is estimated iteratively dropping nonsignificant terms from the model definition, and finally only significant terms remain in the model. The stepwise selection model is similar to the forward model except that such effects are not necessarily to remain in the model. Effects are entered into and removed from the model in such a way that each forward selection step may be followed by one or more backward elimination steps (SAS 2004). This procedure allows us to select the most important contributions responsible for deviation of blood counts, and to keep only those diseases which make significant contributions to the model.

When the best predictor, $d_{\text {best }}$, is found for the considered blood cell line, the regression formula provides a model for frequency of the developing respective cytopenic states versus $d_{\text {best }}$ by rewriting the formula (1) as

$\log \frac{1-f\left(d_{\text {best }}\right)}{f\left(d_{\text {best }}\right)}=\bar{\beta}_{0}+\bar{\beta}_{b} d_{\text {best }}$,

where $\bar{\beta}_{b}$ is the estimated parameter for $d_{\text {best }}$ and $\bar{\beta}_{0}$ includes all other terms in which means of continuous characteristics (such as age) and prevalences of considered diseases are used for predictor variables. From this formula the probability is obtained as

$f\left(d_{\text {best }}\right)=\frac{1}{1+\exp \left(\bar{\beta}_{0}+\bar{\beta}_{b} d_{\text {best }}\right)}$.

Thus, the chosen model provides the logistic or sigmoid shape for the dose-response effect. According to ICRP, in general, the dose-frequency relationship is sigmoid in shape when plotted on linear axes (ICRP 1991, p. 102), therefore Eq. 2 exactly corresponds to the expected shape of the frequencies for deterministic effects.

The estimated model for $p\left(d_{\text {best }}\right)$ possesses two important and useful properties. First, it should represent a proper fit for the frequencies $f\left(d_{\text {best }}\right)$, and second, it allows us to define the notion of the rate of cytopenic state for a control [i.e., as $f_{0}=f\left(d_{\text {best }}=0\right)$ ], and, therefore, to introduce a notion of a dose rate inducing 2-fold increase (TFI) in the frequency of cytopenic states relative to the control. The formula for calculation of the TFI dose rate, $d_{\mathrm{TFI}}$, is obtained by solution of the respective equations (2),

$d_{\mathrm{TFI}}=\frac{\log \left(\left(\exp \left(\bar{\beta}_{0}\right)-1\right) / 2\right)-\bar{\beta}_{0}}{\bar{\beta}_{b}}$.

Alternatively, $d_{\mathrm{TFI}}$ can be expressed in terms of cytopenia frequency of unexposed population,

$d_{\mathrm{TFI}}=\frac{1}{-\bar{\beta}_{b}} \log \frac{2-2 f_{0}}{1-2 f_{0}}=\frac{\log (2)+f_{0}+3 / 2 f_{0}^{2}}{-\bar{\beta}_{b}}+o\left(f_{0}^{2}\right)$.

The series expansion provides a simple formula for estimating $d_{\mathrm{TFI}}$ in $\mathrm{mGy}$ :

$d_{\mathrm{TFI}}($ in $\mathrm{mGy}) \approx \frac{693+10 \cdot f_{0}(\mathrm{in} \%)}{-\bar{\beta}_{b}}$,

where the estimate of the effect of the best predictor in y/Gy is presented in Tables 1, 2, and 4, and the value of 693 is derived from $1000 \cdot \log (2) \approx 693$. This formula shows that $d_{\mathrm{TFI}}$ is basically defined by dose-rate effect $\bar{\beta}_{b}$, and, to a much lesser extent, by the spontaneous frequency $f_{0}$. Standard error for the estimate of $d_{\text {TFI }}$ is calculated by propagating estimated errors for intercept $\bar{\beta}_{0}$ and the doserate (best predictor) effect $\bar{\beta}_{b}$. The latter is small for majority of estimates, so the standard error for $d_{\mathrm{TFI}}$ is determined by those of the dose-rate effect $\bar{\beta}_{b}$. 
The estimation of the TFI dose rate based on the logistic model is stable and advantageous in several respects. The calculation requires only assumptions about logistic type of the shape for dose-response effects typical of deterministic effects. The estimated model provides both the estimate of spontaneous level (or control level) of cytopenia and the estimate of the TFI dose rate without additional assumptions. In this respect the estimate of spontaneous level of cytopenia is stable, because it is obtained by extrapolating the biologically motivated model to the region of zero dose rates rather than by using artificial parameters defining the group of external or internal control (e.g., groups of individuals who received doses below the permissible limit).

\section{Results}

The empiric distributions of each blood count in different RBM dose-rate groups (less $15 \mathrm{mGy} / \mathrm{y}, 15-45 \mathrm{mGy} / \mathrm{y}$, more $45 \mathrm{mGy} / \mathrm{y}$ ) are presented on the Fig. 1. Medians, modes, and means of these distributions are lower for groups with higher dose rates, and these differences are significant.

The dose rate is the only predictor for erythrocytes and thrombocytes, while the set of predictors for other cell lines includes attained age as well. The top part of Table 1 summarizes estimates of parameters of distributions of the cellularity of all blood cell lines of interest. The intercept value characterizes the cellularity of each blood count for people with zero RBM dose rate and zero age (or any age for the model of erythrocytes and thrombocytes). Cellularity at zero doze rate and any age $\bar{a}$ are given as $u+\beta_{a} \bar{a}$. Absolute values of influencing factors $\beta_{r}$ characterize the value of depression of hematopoietic cell lines when the dose/dose rate increases by $1 \mathrm{~Gy}$ and age increases by 1 year. The relative units (absolute value of parameter divided by the value of intercept) are used for a comfortable comparison of estimated parameters.

Then, the frequencies of cytopenic states were investigated using the logistic model. It was found that the set of predictors for frequencies of cytopenic states was the same as in models of respective blood counts. Annual dose rates serve as predictors for thrombocytopenia and neutropenia, and dose rate and age for other cytopenic states. The effect of IR in both these analyses is presented in Table 1. A comparison of the values between cell lines shows that leukocytes (viz, lymphocytes and monocytes) and thrombocytes are the most radiosensitive blood cell lines.

The effect of annual dose rate measured in the year of blood count measurements can be nonlinear. At very high dose rate (i.e., above $150 \mathrm{mGy} / \mathrm{year}$ ) we observed the effect of leveling-off and even decrease in the dose-rate pattern of cytopenia frequencies in all blood cell lines 
Table 2 Variations of dose-rate effect on cellularity of blood counts

\begin{tabular}{ll}
\hline Blood cell line & Dose-rate parameter \\
\hline Leukocytes & Mean of dose rates for two prior years, including the year of measurement \\
Neutrophils & Mean of dose rates for three prior years, including the year of measurement \\
Lymphocytes & Dose rate for the year of measurement \\
Monocytes & Mean of dose rates for three prior years, including the year of measurement \\
Trombocytes & Mean of dose rates for three prior years, including the year of measurement \\
Erythrocytes & Dose rate for the year prior to the year of measurement \\
\hline
\end{tabular}

Fig. 1 Distribution of cellularity displayed by blood counts of people chronically exposed to ionizing radiation in the period of maximal exposure (1950-1956)

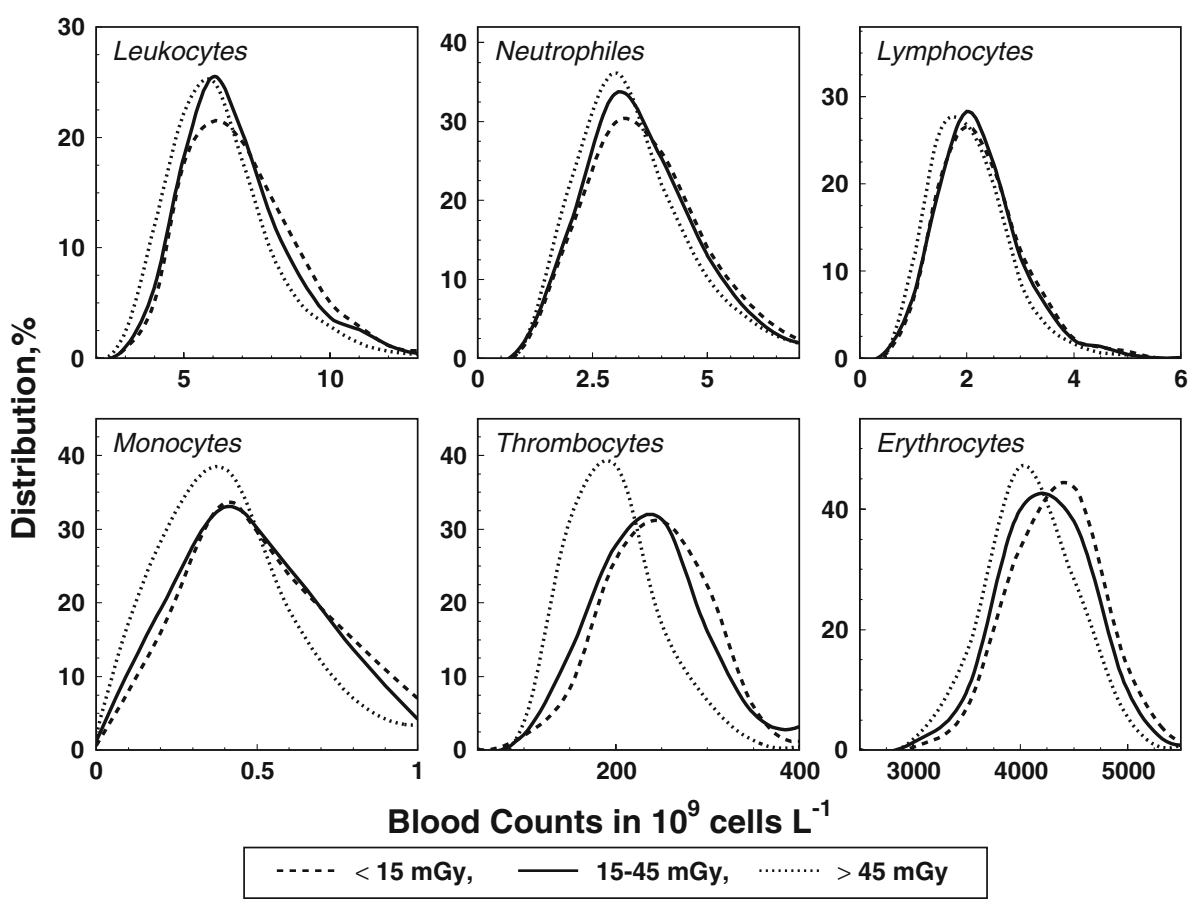

except lymphocytes. This allowed us to hypothesize the existence of some lag period in the effect of exposure. One consequence of this hypothesis is that annual dose rate measured in the year of blood count measurements may be not the only predictor of blood count decline. Instead of using this dose characteristic as the only predictor, we investigate a set of predictors of the frequencies of cytopenia and test respective hypotheses on identifying the best predictor for each blood cell line. Alternative dose predictors were as follows: (1) dose rates measured in years prior to the year of blood count measurements and (2) the means of dose rates over the period prior to the year of blood count measurements. The best predictor represents certain compromise between the effects of instantaneous dose rates and cumulative dose. Because of high correlation between dose rate and cumulative dose [correlation coefficient is $0.53(p<0.0001)$ ], their effects can hardly be interpreted in separate. Using the logistic models and the likelihood ratio test we identified the best dose predictor for each blood cell line as summarized in Table 2. As can be seen from the table, the influence of the previous exposure on response of peripheral blood parameters was obvious for all cell lines, except for the lymphoid cell line. Leukocyte count during prolonged exposure is influenced by the mean of dose rates of two last years, including the year of measurement. Similarly, the thrombocyte, granulocyte, and monocyte counts are dependent on the mean of dose rates of three last years, including the year of measurement. The influence of dose rate on the erythrocyte count in the year previous to the year of measurement was observed. The response of lymphocyte count to exposure was determined by the influence of dose rate in the year of measurement. This type of reaction of peripheral blood cellularity can be explained by the effect of cumulative dose of chronic radiation exposure.

Dependence of frequency of cytopenia on the best dose predictor is shown in Fig. 2. A gradual increase in the frequency of cytopenia with dose-rate value is evident. The logistic model prediction expressed by a simplest version of Eq. 2, i.e., when $\bar{\beta}_{0}=u$, is also shown. 
Fig. 2 Frequencies of cytopenic states for five blood cell lines and leukocytes versus the dose rate identified as the best predictor. Means of frequency estimates are denoted by dots, and standard errors by bars. The solid line corresponds to the model prediction. The dose rate was categorized according to the percentiles of its distribution excepting higher dose rates for which the lower number of measurements was acceptable

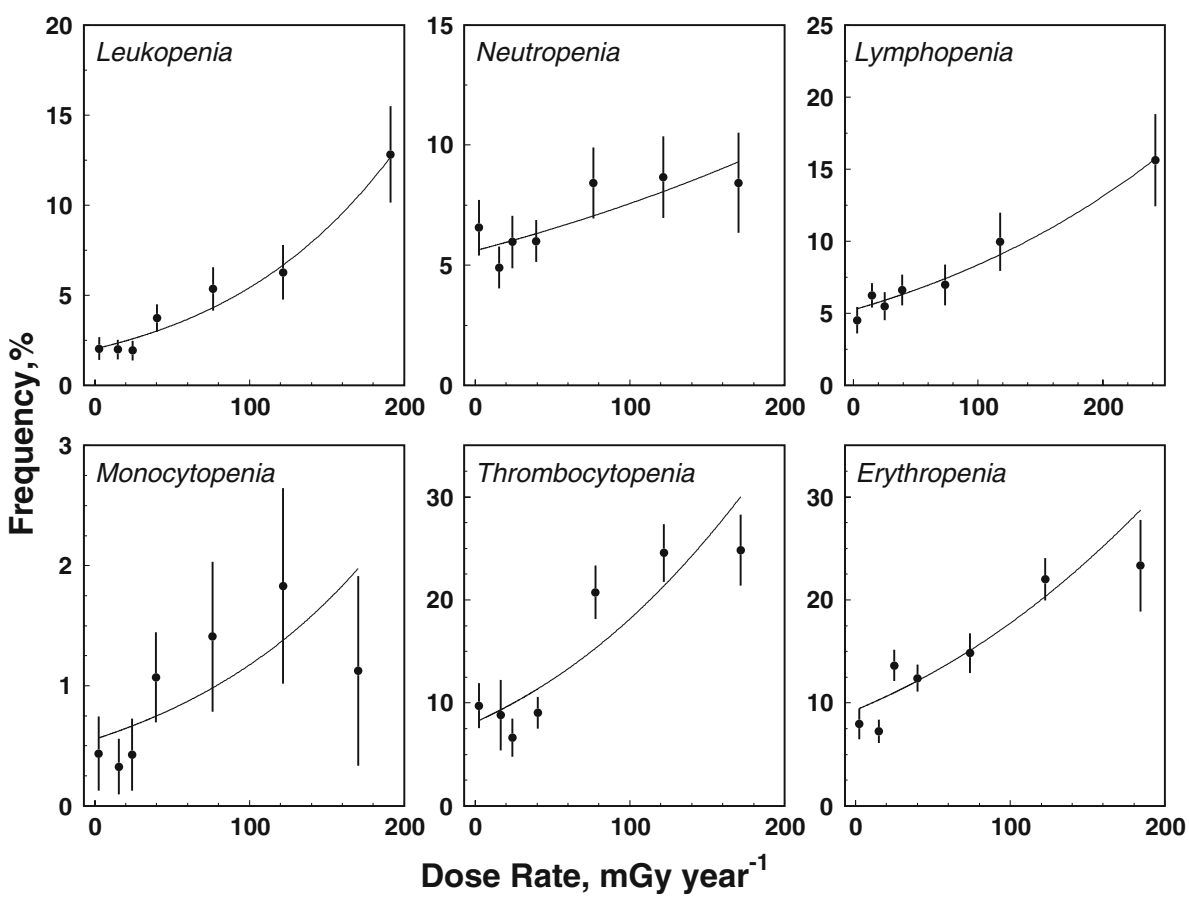

The model taking into account the best dose predictor and the age can be naturally extended to include other information available in the data set, i.e., to take into account the influence of nonradiation factors (age, gender, and health status) on the cytopenia frequency. Respective generalized model is presented by Eq. 1. This model was estimated using the stepwise technique in the logistic regression model allowing for keeping only significant contributions of specific diseases. As a result, we selected 13 clinical conditions whose contribution to the effect on blood counts is significant, and evaluated their effects. The list of the diseases/conditions included brucellosis, flu/ pneumonia, hepatitis/cirrhosis, and other, as well as normal pregnancy. Table 3 presents the set of factors contributing to the model which significantly define the respective frequency.

The data shown in the table characterize the influence of each factor on the probability of cytopenia development in the corresponding blood cell line: the lower the estimated value of the effect exerted by different factors, the higher the probability of cytopenia development. Note that because of using the best dose predictor rather than the dose rate at the year of measurements, the estimates of the respective dose-rate effects presented in Tables 1 and 3 are different, and the effect of the best predictor is stronger. The influence of infectious (brucellosis, trachoma, influenza), parasitic (protozoal intestinal diseases) diseases, chronic noninfectious diseases (anemias, chronic hepatitis, cirrhosis, nephritis, nephritic syndrome), and normal pregnancy has been revealed following an increase in the frequency of cytopenia. The degree of radiation exposure influence prevails under the influence of other modifying factors.

It is rather difficult to apply ICRP criteria (ICRP 1984) to threshold dose estimation for cytopenia induction during chronic radiation exposure because of the spontaneous level of cytopenia [for the Russian population, the level of leukopenia is $2.0-5.2 \%$, erythropenia for men is $4.0 \%$, for women $5.1 \%$, thrombocytopenia is $2.2 \%$ (Sokolov et al. 1985)]. An important property of the logistic modeling applied is not only that it represents a proper fit for the frequencies, but that it also allows us to estimate the frequency of cytopenic states for controls (i.e., the frequency for a zero dose rate), and, therefore, to introduce a notion of the dose rate associated with a 2 -fold increase in the frequency of cytopenia. By the TFI dose rate we understand such a dose rate for which the rate of cytopenic states is increased by a factor of two compared to the control. The TFI dose rate $\left(d_{\mathrm{TFI}}\right)$ can be considered as a quantitative characteristic of the effects of chronic exposure.

The TFI dose rates calculated using the approach based on the logistic model for members of TRC are presented in Table 4 . The calculation of $d_{\mathrm{TFI}}$ was performed taking into account the age structure of the population, i.e., assigning members of the study population to a specific age group was considered as an explanatory variable. This allows for extrapolating results for other populations with different age structure.

Judging by TFI dose-rate parameter, the thrombocyte line $(84 \mathrm{mGy} / \mathrm{year})$ is the most radiosensitive one. The frequency of leukopenia increased 2-fold at the dose rate of $56 \mathrm{mGy} / \mathrm{year}$, and that of erythrocytopenia at $103 \mathrm{mGy} /$ 


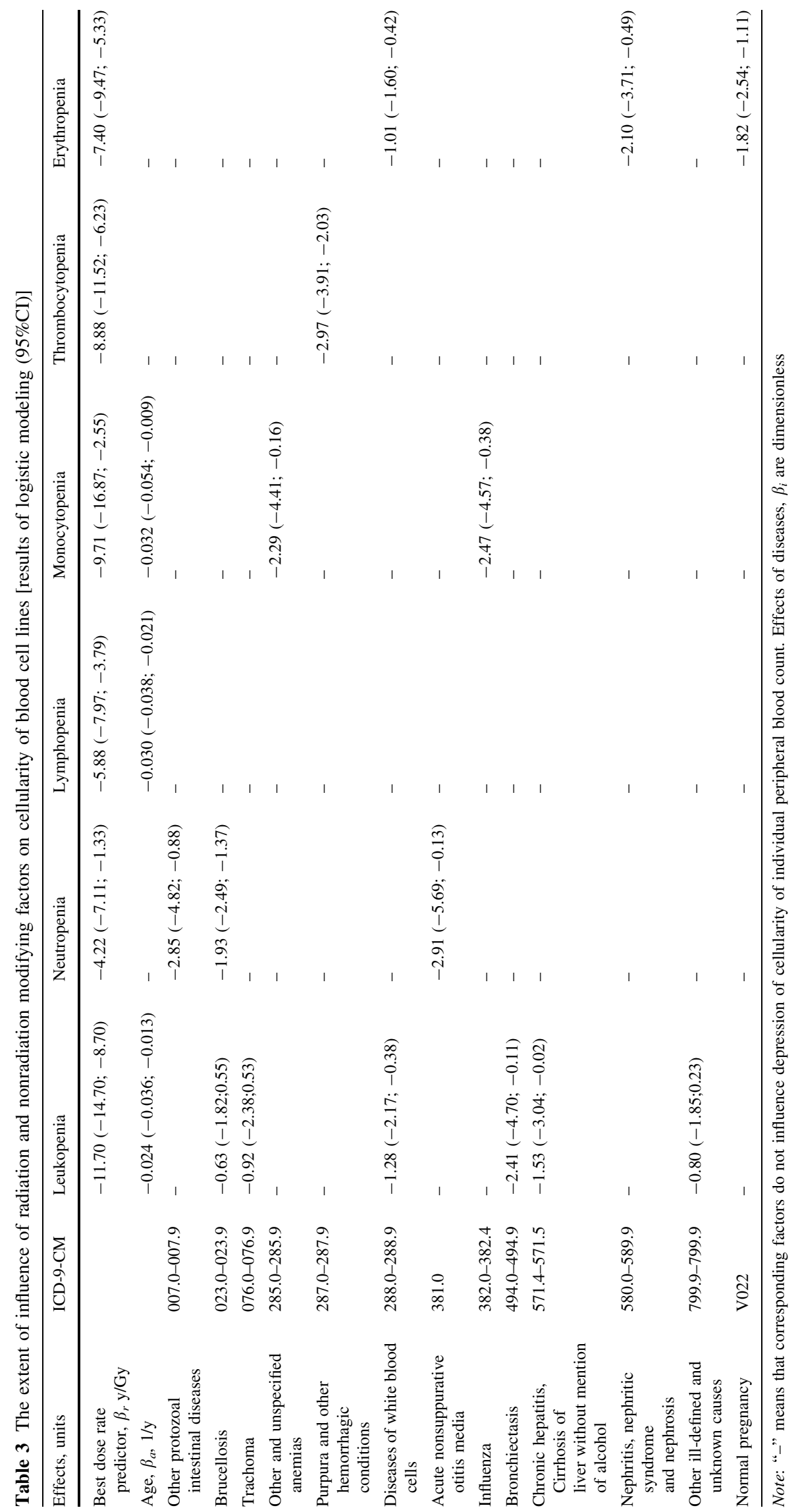


Table 4 The estimated dose rate inducing TFI in cytopenia frequency (mGy/year) with standard error or $95 \%$ confidential intervals

\begin{tabular}{lllc}
\hline Blood count & Dose-rate effect, $\bar{\beta}_{b}, \mathrm{y} / \mathrm{Gy}$ & Frequency $f_{0}, \%$ & TFI dose rate, mGy/y \\
\hline Leukocytes & $-12.5 \pm 1.5$ & $1.5(1.1,2.0)$ & $56.8 \pm 7.0$ \\
Neutrophils & $-3.6 \pm 1.5$ & $5.3(4.3,6.4)$ & $206.7 \pm 87.8$ \\
Lymphocytes & $-6.2 \pm 1.1$ & $4.4(3.6,5.4)$ & $118.9 \pm 20.8$ \\
Monocytes & $-8.7 \pm 1.3$ & $0.6(0.5,0.7)$ & $86.2 \pm 12.9$ \\
Trombocytes & $-9.2 \pm 1.4$ & $8.1(6.3,10.4)$ & $84.2 \pm 12.7$ \\
Erythrocytes & $-7.5 \pm 1.1$ & $8.8(7.6,10.3)$ & $103.4 \pm 15.0$ \\
\hline
\end{tabular}

year. When the neutrophiles and lymphoid lines are estimated separately, it can be seen that the value of dose rate, which leads to a 2-fold increase in the frequency of cytopenias for the lymphoid cell line increases up to $118.9 \mathrm{mGy} / \mathrm{year}$, and that for neutrophiles-up to $206.7 \mathrm{mGy} / \mathrm{year}$. This parameter is estimated for gender and age groups. Application of this approach to specific population groups and calculation of respective TFI dose rates showed that compared to men, women had a slightly higher radiosensitivity, as is demonstrated by the example of the frequency of neutropenia and lymphopenia. Exposed people aged 55 years or older at the start of exposure also demonstrated a higher radiosensitivity.

\section{Discussion}

Hematopoiesis inhibition is considered to be the main early effect of chronic low-dose-rate radiation exposure, which is manifested by depression of RBM hematopoiesis and cellularity of peripheral blood (Akleyev and Varfolomeyeva 2007; Fliedner and Graessle 2008). Complete blood count is the most accessible and widely used method to evaluate the status of hematopoiesis (Sokolov et al. 1985; Dainiak et al. 2003). Mathematical methods promote identification of the radiation-induced modifying factors of changes in hematopoiesis and the most important characteristics of exposure affecting the parameters of cellularity of peripheral blood.

In the course of investigation the dose-rate parameter was found to be a determinant factor for changes in peripheral blood cellularity observed among people exposed on the Techa River. The tension of regenerative processes in RBM is confirmed by the influence of previous exposure (effect of cumulative dose) on cellularity of peripheral blood which continues for several years in different blood cell lines. Monitoring of thrombocyte, granulocyte, and monocyte counts revealed a latent period in the reaction of these blood cell lines to chronic radiation exposure.

Mature forms of blood cells (except lymphocytes) are rather radioresistant, while stem and progenitor cells are highly radiosensitive (Fliedner et al. 2002b). Accumulation of DNA damage requiring repair under chronic radiation exposure leads to a gradual decrease in proliferating activity of hematopoietic precursors (Seed and Meyers 1993). At the same time, the cell's ability for repair is reduced too (Plappert et al. 1997). A slow exhaustion of compensation can be observed in actively proliferating cell compartments (CFU-GEMM and earlier) (Fliedner et al. $2002 \mathrm{~b}$ ). The fact that these cells undergo active phases of cell cycle leads to an increase in their radiosensitivity and, consequently, to incurrence of damage (Cheng 2004; Valerie et al. 2007). Decrease in proliferative activity of hematopoietic precursors results in reduction in their mature forms in peripheral blood (Fliedner and Graessle 2008).

The situation in the erythroid cell lineage has some peculiarities. The influence of dose rate in the year previous to the year of erythrocyte counts on the erythroid line cellularity is indicative of a more manifest compensatory ability of this blood cell line. It is well known that a decrease in RBM erythroblasts under chronic radiation exposure is compensated by a shift of stem cell differentiation to the red cell line (Gruzdev et al. 1963). The data presented by (Akleyev and Varfolomeyeva 2007) show that the leading role in sustaining the normal level of erythrocytes in the peripheral blood under chronic radiation exposure is played by the proliferative activity of erythropoietic cell precursors. This is also confirmed by the elevation of erythrocaryocyte level in RBM, and the level of mitosis in the line.

Lymphoid cell line has some peculiarities in comparison with other counts. The reaction of lymphoid cells is rather rapid (without any delay) in the case of chronic radiation exposure because they are the most radiosensitive blood cells (both young and mature forms) (Safwat 2000; Tuschl et al. 1995).

Obviously, lymphoid cells have considerable capacity for migration; maturation and differentiation of lymphocytes take place in the thymus and lymphatic nodes (Woodruff et al. 1987; Fliedner et al. 2002b). These organs are to a lesser extent influenced by ${ }^{90} \mathrm{Sr}$ incorporated in bones. They are exposed for a very brief time period to 
gamma irradiation (mean dose rate was $92 \mathrm{mGy} / \mathrm{y}$, maxi$\mathrm{mal}-206 \mathrm{mGy} / \mathrm{y}$ ) from 1950 to 1952 (Degteva et al. 2006).

As was mentioned above, the existing principles of threshold estimation of deterministic effects suggested by ICRP (1984) are more applicable for cases of acute radiation exposure and are not suitable for chronic radiation exposure of low intensity. In such situations, the frequency of cytopenia can vary under simultaneous influence of concomitant diseases. This increases the level of spontaneous cytopenia in the exposed population. The estimation of influence and ranging of nonradiation factors which increase the frequency of cytopenic states helps reveal the prevailing factor. The TFI dose-rate parameter allows taking into account the changing level of spontaneous (nonradiation) cytopenia and demonstrates reliably an increase in its frequency with dose rate. The dose leading to a 2-fold increase in cytopenia in the investigated blood cell line can also be considered as a characteristic of radiosensitivity.

People of older age chronically exposed to radiation showed an increase in radiosensitivity of lymphoid and erythroid lines of hematopoiesis. Progenitor cell pool which plays the role of a pluripotent precursor reserve decreases with age (Bagnara et al. 2000). The ability of the progenitor pool to self-maintenance diminishes too (Marley et al. 1999). Chronic radiation exposure as a stress factor can lead to different degrees of hematopoietic insufficiency manifested by elderly people.

Modeling of changes in hematopoiesis for exposed general populations (Russia, Germany, United States of America), including men and women of different ages, has been conducted with the purpose to test this approach (Table 5). These estimations showed that the 2-fold increase in the dose-rate parameter for different blood counts made for the populations of the USA, Germany, and Russia is stable and applicable for populations differing in age and gender structure.

Table 5 Dose rate for TFI in cytopenia frequency (mGy/year) estimated for three different countries

\begin{tabular}{lccc}
\hline Blood count & USA (2006) & Germany (2006) & Russia (2002) \\
\hline Leukocytes & 56.7 & 56.8 & 56.7 \\
Neutrophils & 205.6 & 205.4 & 205.7 \\
Lymphocytes & 119.2 & 120.1 & 119.5 \\
Monocytes & 86.4 & 86.4 & 86.1 \\
Trombocytes & 84.3 & 84.1 & 84.1 \\
Erythrocytes & 104.0 & 103.6 & 103.6
\end{tabular}

Note: The year of status of age- and gender-structure of the populations of the 3 countries is indicated in brackets (Human Mortality Database (HMD) 2009; The All-Russia Population Census 2002)

\section{Conclusions}

It has been demonstrated in this study that a long-term (over many years) low-dose rate exposure to IR (maximal dose rate reaches $0.3 \mathrm{~Gy} / \mathrm{y}$ ) results in inhibition of hematopoiesis manifested by decreased cellularity of peripheral blood. The intensity of inhibition is determined by the dose rate measured in (or prior to) the year of measurement and is largely related to the cumulative character of the effect and determined by the dose rates measured 1 or 2 years before the blood counts were made (the influence of cumulative dose). The impact of specific individual characteristics (e.g., attained age, age at maximal exposure, health state represented by ICD-9 diagnoses, and demographic characteristics) has been evaluated and shown to be a much less important factor contributing to hematopoiesis inhibition than the dose characteristics. The value of 100-200 mGy/year is interpreted as a level of a 2-fold increase in hematopoiesis inhibition. This parameter is considered as a characteristic of quantitative description in the case of chronic exposure to IR.

Thus, a series of findings highlighting a number of features of early effects on hematopoiesis in chronic radiation exposure have been obtained as result of the studies presented in this paper. Further studies are needed to develop an unambiguous view on the mechanisms of development of early effects under chronic exposure to IR, and to relate them to the late ones, and to reveal, if possible, their cause-effect relationship. A number of specific points which require further analyses deal with the following questions: (1) what is the potential for adaptation characteristic of the hematopoietic system affected by chronic exposure to IR of low- and intermediate-dose range? (2) what is the pattern of the relationship between early and late effects manifested by the hematopoietic system (3) to what extent are these effects deterministic and how can the influence of population heterogeneity on these effects be quantified? (4) what residual changes in the hematopoietic system persist in the long run at molecular, cell, and tissue levels and what is their role in the development of IR-induced leukemia? and (5) what influence is exerted by exposure dose, dose rate, and duration of RBM exposure on long-term status of hematopoiesis? These important questions have to be addressed in further studies.

Conflict of interest statement The authors declare that they have no conflict of interest.

\section{References}

Akleyev A, Varfolomeyeva T (2007) Dynamics of blood cell composition in residents of the Techa riverside villages. Acta Med Nagasaki 52:19-28 
Akleyev AV, Veremeyeva GA, Silkina LA, Vozilova AV (1999) Long-term hemopoiesis and immunity status after chronic radiation exposure of red bone marrow in humans Centre. Eur J Occup Environ Med 5:113-129

All-Russia Population Census (2002) Basic result. Federal State Statistics Service. http://www.perepis2002.ru/index.html?id=86. Accessed Nov 2008

Anspaugh LR, Degteva MO, Vorobiova MI (2006) Dosimetry for members of the extended Techa River cohort. Health Phys 91:393-394

Bagnara GP, Bonsi L, Strippoli P et al (2000) Hemopoiesis in healthy old people and centenarians: well-maintained responsiveness of CD34 + cells to hemopoietic growth factors and remodeling of cytokine network. J Gerontol A Biol Sci Med Sci 55:61-70

Baraboř VA, Oleňnik SA (1999) Stress in the etiology of radiation injury. Role of regulatory mechanisms. Radiats Biol Radioecol 39:438-443 (in Russian)

Bond VP, Fliedner TM, Archambeau JO (1965) Mammalian radiation lethality: a disturbance in cellular kinetics. Academic Press, New York, London

Cheng T (2004) Cell cycle inhibitors in normal and tumor stem cells. Oncogene 23:7256-7266

Cox DR, Hinkley DV (1974) Theoretical statistics. Chapman and Hall, London

Dainiak N, Waselenko JK, Armitage JO et al. (2003) The hematologist and radiation casualties. Hematol Am Soc Hematol Educ Program 473-496

Degteva MO, Vorobiova MI, Tolstykh EI (2006) Development of an improved dose reconstruction system for the Techa River population affected by the operation of the Mayak Production Association. Radiat Res 166:255-270

Degteva MO, Shagina NB, Tolstykh EI et al (2007) An approach to reduction of uncertainties in internal doses reconstructed for the Techa River population. Radiat Prot Dosimetry 127:480-485

Fliedner TM, Graessle DH (2008) Hematopoietic cell renewal systems: mechanisms of coping and failing after chronic exposure to ionizing radiation. Radiat Environ Biophys 47:6369

Fliedner TM, Feinendegen LE, Hopewell JW (2002) Chronic irradiation: tolerance and failure in complex biological systems. Br J Radiol Suppl 26, London

Fliedner TM, Graessly D, Paulsen C, Reiners K (2002b) Structure and function of bone marrow hemopoiesis: mechanisms of response to ionizing radiation exposure. Cancer Biother Radiopharm $17: 405-426$

Gruzdev GP, Yevseyenko NK, Rozhdestvensky LM et al (1963) Failure of cell regeneration of red bone marrow in rats exposed to ionizing radiation. Med Radiol 8:35-42 (in Russian)

Grygoryev YuG, Popov VI, Shifirkin AV, Antipenko DB (1986) Somatic effects of chronic exposure by gamma irradiation. Energoatomizdat, Moscow

Guskova AK, Baysogolov GD (1971) Radiation sickness of human. Meditsina, Moscow (in Russian)

Hosmer D, Lemeshow S (2000) Applied logistic regression. John Wiley, New York

Human Mortality Database (HMD) (2009) University of California at Berkeley, Max Planck Institute for Demographic Research. http://www.mortality.org. Access Jan 2009

ICRP (1984) International Commission on Radiological Protection, Publication 41: nonstochastic effects of ionizing radiation. Pergamon Press, Oxford

ICRP (1991) International Commission on Radiological Protection, Publication 60. Pergamon Press, Oxford
Kossenko MM, Thomas TL, Akleyev AV et al (2005) The Techa River Cohort: study design and follow-up methods. Radiat Res 164:591-601

Marley SB, Lewis JL, Davidson RJ et al (1999) Evidence for a continuous decline in haemopoietic cell function from birth: application to evaluating bone marrow failure in children. $\mathrm{Br} \mathrm{J}$ Haematol 106:162-166

McCullagh P, Nelder JA (1989) Generalized linear models, 2nd edn. Chapman and Hall, London

Muksinova KN, Mushkacheva GS (1990) Cell and molecular basis of hemopoiesis rearrangement during protracted radiation exposure. Energoatomizdat, Moscow

Napier BA, Shagina NB, Degteva MO, Tolstykh EI, Vorobiova MI, Anspaugh LR (2001) Preliminary uncertainty analysis for the doses estimated using the Techa River dosimetry system-2000. Health Phys 81:395-405

Novoselova EG, Safonova MV (1994) Functional activity of spleen Tand B-lymphocytes of rats exposed to chronic low-dose gamma radiation. Radiats Biol Radioecol 34:407-413 (in Russian)

Okladnikova ND (2001) In: Ilyin LA (ed) Radiation medicine. Izdat, Moscow, pp 253-274 (in Russian)

Paranich AV, Tyslenko KV, Frolova NA et al (2001) Structural and functional changes in various cells following radiation exposure. Biofizika 46:1103-1107 (in Russian)

Plappert UG, Stocker B, Fender H, Fliedner TM (1997) Changes in the repair capacity of blood cells as a biomarker for chronic lowdose exposure to ionizing radiation. Environ Mol Mutagen $30: 153-160$

Safwat A (2000) The immunobiology of low-dose total-body irradiation: more questions than answers. Radiat Res 153:599-604

SAS (2004) SAS 9.1. Help and documentation. SAS Institute Inc., Cary

Seed TM (1996) Hematopoietic tissue repair under chronic low daily dose irradiation. Adv Space Res 18:65-70

Seed TM, Meyers SM (1993) Chronic radiation-induced alteration in hematopoietic repair during preclinical phases of aplastic anemia and myeloproliferative disease: assessing unscheduled DNA synthesis responses. Cancer Res 53:4518-4527

Seed TM, Tolle DV, Fritz TE (2002) Haematological response to chronic irradiation: the past Argonne experience and future AFRRI initiatives. BJR Suppl 26: 94-103

Seed TM, Fritz TE, Tolle DV, Jackson WE (2002b) Hematopoietic responses under protracted exposures to low daily dose gamma irradiation. Adv Space Res 30:945-955

Shvedov VL, Akleyev AV (2001) Radiobiology of strontium-90. Megas, Chelyabinsk (in Russian)

Sokolov VV, Gribova IA (1972) Hematological parameters of healthy man. Meditsina, Moscow (in Russian)

Sokolov VV, Gribova IA, Vyalova NA, Suvorova LA (1985) Blood and bone marrow investigation. In: Ilyin LA (ed) Organization of medical follow-up of exposed population. Energoatomizdat, Moscow, pp 19-41 (in Russian)

Tuschl H, Steger F, Kovac R (1995) Occupational exposure and its effect on some immune parameters. Health Phys 68:59-66

Valerie K, Yacoub A, Hagan MP et al (2007) Radiation-induced cell signaling: inside-out and outside-in. Mol Cancer Ther 6:789-801

Woodruff JJ, Clarke LM, Chin YH (1987) Specific cell-adhesion mechanisms determining migration pathways of recirculating lymphocytes. Annu Rev Immunol 5:201-222

Yagunov AS, Tokalov SV, Chukhlovin AB, Afanassiev BV (1998) Animal studies of residual hematopoietic and immune system injury from low dose/low dose rate radiation and heavy metals. Armed Forces Radiobiology Research Institute, Bethesda 